\title{
Simple applications of continued fractions and an elementary result on Heron's algorithm
}

\author{
Antonino Leonardis \\ Department of Mathematics and Computer Science, Universit della Calabria \\ Arcavacata di Rende, Italy \\ e-mail: a.leonardis@gmail.com
}

Received: 15 January 2018 Revised: 22 September $2018 \quad$ Accepted: 26 October 2018

\begin{abstract}
The paper is a continuation of the author's previous works on continued fractions, and has been presented at the AMS special session on Continued Fraction during the Joint Mathematical Meetings 2017, Atlanta GA. The first part is more introductory/educational, explaining the importance of matricial and Diophantine methods in the topic of continued fractions. We will begin this part discussing geometrical illusions which can arise from properties of continued fractions and associated matrices, proving thoroughly the mathematical reasons of this fact. After this, we will deal with the Pythagorean problem of the right-angled isosceles triangles finding all solutions to the simple Diophantine equation $l^{2}+(l+1)^{2}=d^{2}$, which will give a "PseudoPythagoric" triangle. In the second part, recalling all the methods introduced in the first one, we will prove a theorem (the main result), which relates continued fractions with Heron's algorithm, giving some examples. This theorem is proved in a complete form, which considers all possibilities and its vice-versa, unlike all other minor results that can be found in the literature (see [2,3]). Keywords: Continued fractions, Heron's algorithm, Matrices, Fibonacci sequence, Pythagorean triples, Diophantine equations.
\end{abstract}

2010 Mathematics Subject Classification: 11A55, 11C20, $11 \mathrm{D} 09$.

\section{Introduction}

The theory of continued fractions in the field of real numbers is very interesting as well as full of applications. The literature deals widely with this topic, for example in the books mentioned 
in the bibliography (Cassels [1], Perron [4]). A continued fraction, in our case, will be a nested fraction of the form:

$$
n_{0}+\frac{1}{n_{1}+\frac{1}{n_{2}+\frac{1}{n_{3}+\cdots}}}=:\left[n_{0}, n_{1}, \ldots\right]
$$

with the following conditions on the "digits" $\left\{n_{i} \in \mathbb{Z}\right\}_{i \in I \subseteq \mathbb{N}}$ :

$$
\forall i>0: n_{i}>0
$$

The continued fraction expansion will be called finite if the $n_{i}$ are finitely many. Finite continued fractions represent all rational numbers in exactly two ways, where one is obtained by the other by writing the last digit $n_{k}$ as $\left(n_{k}-1\right)+\frac{1}{1}$ (thus substituting it with the two digits $\left.n_{k}^{\prime}=\left(n_{k}-1\right), n_{k+1}^{\prime}=1\right)$.

On the other hand, when $n_{i}$ is a full sequence of integers $(I=\mathbb{N})$, the continued fraction is called infinite. Despite being indefinitely long, it always converges (or, more precisely, the sequence of its finite truncations converges) to a limit $\xi \in \mathbb{R}$, and - vice versa - any irrational $\xi \in \mathbb{R} \backslash \mathbb{Q}$ is represented by a unique infinite continued fraction. One may ask what happens when "digits" periodically repeat themselves or when they do not; in this case the well-known Lagrange's theorem ensures that an infinite continued fraction converges to a quadratic irrational if and only if its digits definitely repeat themselves periodically.

The paper will begin with some simple applications of continued fractions, starting with geometric illusions (Fig. 1, 2 and 3) and then solving the Diophantine equation (1) which gives a sequence of "pseudo-isosceles" right-angled triangles, that is, a good approximating solution for the Pythagorean problem of the ratio between the diagonal and the side of a square. These applications also highlight connections with matricial methods and linear fractional transformations of the real line, which are the restriction of a projectivity in $\mathbb{P}_{1}(\mathbb{R})$ given indeed by a (homogeneous) matrix. After this, we will prove a theorem on the relationship between continued fractions and Heron's algorithm (Theorem 4.4). This algorithm is very important in the history of mathematics, and is a particular case of Newton's method for approximating zeroes of a function. This main theorem is an elementary result, but the aim of this paper is to give it an original precise statement and an equally original proof with the already mentioned matricial methods, as well as remarking the necessity and sufficiency of the here given conditions. For more details about the matricial expression of a projectivity, see the Appendix A on this subject.

\section{Geometrical illusions and continued fractions}

We start by seeing a simple classical illusion trick tied to matrix determinants to introduce the world of continued fractions.

\section{1 "Square to rectangle" illusions}

Let's start by recalling the classical Fibonacci illusion (Fig. 1): 


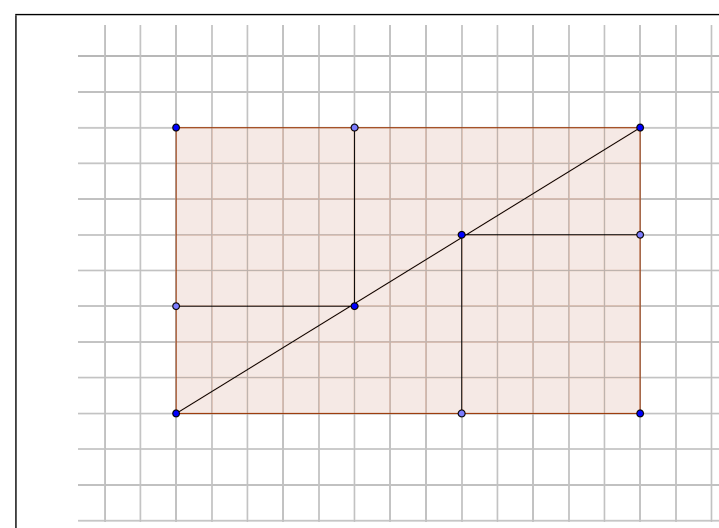

(a) Decomposition of an $8 \times 13$ rectangle apparently using 4 triangles and two $5 \times 5$ squares.

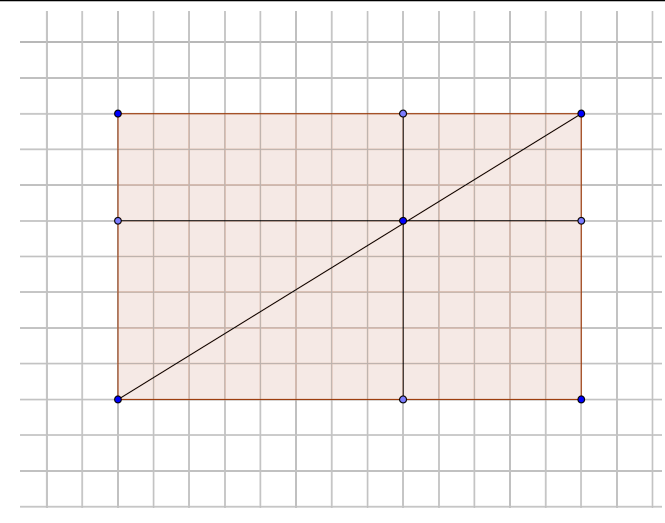

(b) Decomposition similar to the former one, but a $5 \times 5$ square is replaced by a $3 \times 8$ rectangle.

Figure 1. Fibonacci illusion

We see that there is a "missing square" $(5 \times 5=3 \times 8+1)$; this happens because the four points on the diagonal are not actually aligned but instead form a parallelogram with area exactly 1 . Indeed, this area is given by the absolute value of the determinant of a $2 \times 2$ matrix (the one whose columns represent the sides of the parallelogram); in this case we find the matrix via the continued fraction approximation of the golden ratio (see section "atricial methods" below), and the theory of continued fractions ensures us that these matrices have determinant \pm 1 . Approximating the square root of 2 , we get indeed a similar example:

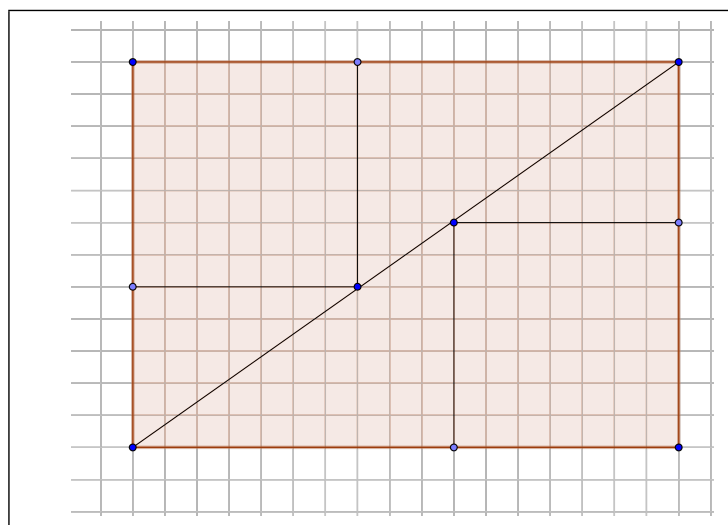

(a) Decomposition of a $12 \times 17$ rectangle apparently using 4 triangles and two $7 \times 7$ squares.

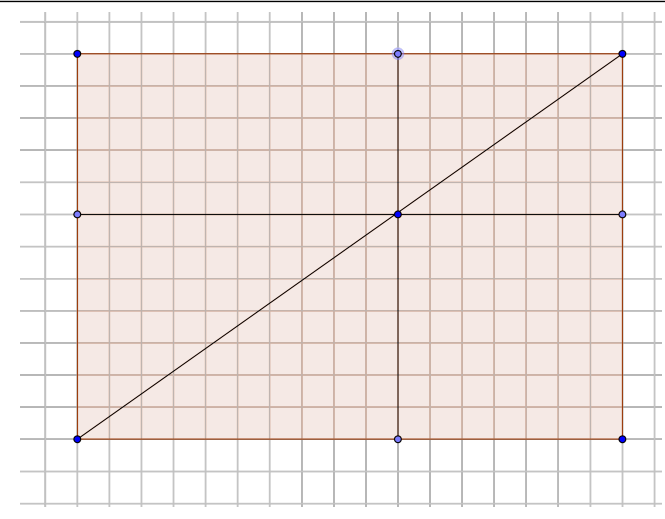

(b) Decomposition similar to the former one, but a $7 \times 7$ square is replaced by a $5 \times 10$ rectangle.

Figure 2. Papersheet illusion

In this case we have $7 \times 7=5 \times 10-1$. We will name the latter papersheet illusion, recalling that the ratio between sides of a common sheet of paper is approximately $\sqrt{2}$.

\subsection{Matricial methods}

See also Appendix A. 
We recall that, considering the (symmetric) matrix $\widehat{a_{0}}=\left(\begin{array}{cc}n_{0} & 1 \\ 1 & 0\end{array}\right)$ as a $\mathbb{P}^{1}$ projectivity (that is, a linear fractional transformation), it is matter of a bit of calculations to prove that $\left[n_{0}, n_{1}, n_{2}, \ldots\right]=$ $\widehat{n_{0}}\left[n_{1}, n_{2}, \ldots\right]$ for every continued fraction.

Indeed, if $x=\left[n_{1}, n_{2}, \ldots\right]=\frac{p}{q}$, we have immediately:

$$
\widehat{n_{0}} \frac{p}{q}=\frac{n_{0} p+q}{p+0 q}=n_{0}+\frac{q}{p}=n_{0}+\frac{1}{x} .
$$

We will associate the $j$-th approximant $\left[n_{0}, n_{1}, \ldots, n_{j-1}\right]$ with the matricial composition (" $a p$ proximant matrix") $\widehat{n_{0}} \cdot \widehat{n_{1}} \cdots \widehat{n_{j-1}}$.

Remark 2.1. The first column of the latter matrix is exactly the homogeneous vector in $\mathbb{P}^{1}$ representing $\left[n_{0}, n_{1}, \ldots, n_{j-1}\right]$. Moreover, the second column is ${ }^{1}$ the same as the first column of $\widehat{n_{0}} \cdot \widehat{n_{1}} \cdots \widehat{n_{j-2}} \cdot I_{2}$, thus represents $\widehat{n_{0}} \cdot \widehat{n_{1}} \cdots \widehat{n_{j-2}}$.

We know that the continued fraction of the golden ratio $\lambda=\frac{\sqrt{5}+1}{2}$ is:

$$
\lambda=[1,1,1, \ldots]=[\overline{1}]
$$

and truncating this expression we get the good approximations ensured by Dirichlet's lemma (see [1]):

$$
\begin{aligned}
{[1] } & \rightarrow\left(\begin{array}{ll}
1 & 1 \\
1 & 0
\end{array}\right) ; \\
{[1,1] } & \rightarrow\left(\begin{array}{ll}
1 & 1 \\
1 & 0
\end{array}\right)^{2}=\left(\begin{array}{ll}
2 & 1 \\
1 & 1
\end{array}\right) ; \\
{[1,1,1] } & \rightarrow\left(\begin{array}{ll}
1 & 1 \\
1 & 0
\end{array}\right)^{3}=\left(\begin{array}{ll}
3 & 2 \\
2 & 1
\end{array}\right) ; \\
{[1,1,1,1] } & \rightarrow\left(\begin{array}{ll}
1 & 1 \\
1 & 0
\end{array}\right)^{4}=\left(\begin{array}{ll}
5 & 3 \\
3 & 2
\end{array}\right) ; \\
{[1,1,1,1,1] } & \rightarrow\left(\begin{array}{ll}
1 & 1 \\
1 & 0
\end{array}\right)^{5}=\left(\begin{array}{ll}
8 & 5 \\
5 & 3
\end{array}\right) ; \\
{[1,1,1,1,1,1] } & \rightarrow\left(\begin{array}{ll}
1 & 1 \\
1 & 0
\end{array}\right)^{6}=\left(\begin{array}{ll}
13 & 8 \\
8 & 5
\end{array}\right) .
\end{aligned}
$$

As we can see, the ratio between the elements of the first column (which is the numerical value of the truncation) is the quotient of two consecutive Fibonacci numbers. The fifth matrix gives us the area of the parallelogram involved in the Fibonacci illusion (as remarked before, by taking the absolute value of its determinant).

\footnotetext{
${ }^{1}$ Indeed, $\left(\begin{array}{l}1 \\ 0\end{array}\right)$ is both the second column of $\widehat{n_{j-1}}$ and the first column of $I_{2}=\left(\begin{array}{ll}1 & 0 \\ 0 & 1\end{array}\right)$.
} 
In the case of $\sqrt{2}=[1,2,2,2, \ldots]=[1, \overline{2}]$ we have similarly:

$$
\begin{aligned}
{[1] } & \rightarrow\left(\begin{array}{ll}
1 & 1 \\
1 & 0
\end{array}\right) ; \\
{[1,2] } & \rightarrow\left(\begin{array}{ll}
3 & 1 \\
2 & 1
\end{array}\right) ; \\
{[1,2,2] } & \rightarrow\left(\begin{array}{ll}
7 & 3 \\
5 & 2
\end{array}\right) ; \\
{[1,2,2,2] } & \rightarrow\left(\begin{array}{ll}
17 & 7 \\
12 & 5
\end{array}\right) ; \\
{[1,2,2,2,2] } & \rightarrow\left(\begin{array}{ll}
41 & 17 \\
29 & 12
\end{array}\right) .
\end{aligned}
$$

Subtracting in the fourth matrix (whose first column gives the sides of the bigger rectangle) the second column from the first we obtain the matrix $\left(\begin{array}{cc}10 & 7 \\ 7 & 5\end{array}\right)$ and the relative small parallelogram of area 1 involved in the papersheet illusion. To have a similar result with the original matrix one should consider a $24 \times 17$ rectangle.

\section{3 "Four triangles" illusions}

A similar illusion can be made with an incorrect decomposition of the big rectangle in four triangles:

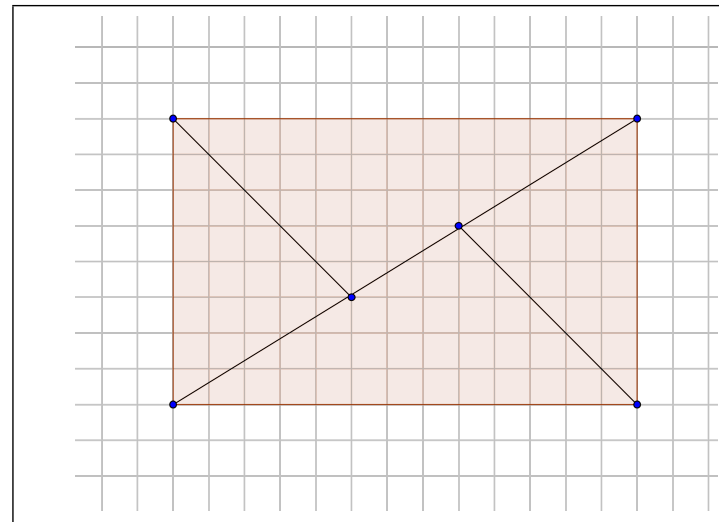

(a) The area of the four triangles seems to be $5 \times 8+5 \times 13=105$ but the whole rectangle has area $8 \times 13=104$.

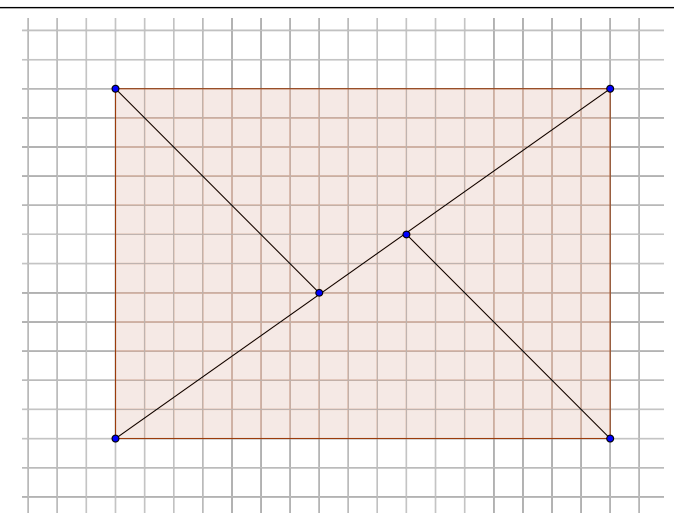

(b) The area of the four triangles seems to be $7 \times 12+7 \times 17=203$ but the whole rectangle has area $12 \times 17=204$.

Figure 3. Triangles illusion

Here the parallelogram issue is even more clear, as the surface in it is exactly the difference between the apparent area of the rectangle and the real one.

\subsection{Generalized illusion}

Summing up, we have: 
Result 2.2. Any $2 \times 2$ matrix with determinant \pm 1 and entries in $\mathbb{N}$, as the ones given by continued fractions' approximation, creates the illusions described above. Indeed, one has to draw the parallelogram with sides given by the column of the matrix, the Cartesian rectangle (i.e., whose sides are aligned with Cartesian axes) with same diagon al as the parallelogram, and complete the figure as we did in the Fibonacci and papersheet case.

More precisely, setting the bottom-left corner of the rectangle as the origin of the Cartesian plane, the two points on the diagonal are given by the two columns of the chosen matrix (considered as vectors in $\mathbb{Z}^{2}$ ), and the whole diagonal is exactly the sum of these two vectors (so that the dimensions of the whole rectangle are given by the coordinates of this vectorial sum).

\section{Pseudo-Pythagorean triangles}

It is well known that there is no isosceles right-angled triangle with integral (or rational) sides. We can though find approximated solutions to this Pythagorean dilemma.

Pythagorean disciples were left astonished discovering that there is no right-angled isosceles triangle with integer (and, consequently, neither rational) sides. Still, as the Pythagoreans did with music tones, we can try to use (Diophantine) approximation. Irrationality of $\sqrt{2}$ cannot be overcome, but still we can look for a sequence of approximating solutions: let us see how.

We will call "Pseudo-Pythagoric triangle" a right-angled one with integer sides such that the two catheti differ by only one unit (that is, almost isosceles). This means solving the Diophantine equation:

$$
l^{2}+(l+1)^{2}=d^{2}
$$

which is equivalent by elementary calculations (or using norm properties for the Gaussian integer $l+i(l+1)$ multiplied by $i+1)$ to $1+(2 l+1)^{2}=2 d^{2}$. Setting $k=2 l+1$, we can reduce to solving the following Pell's equation:

$$
k^{2}-2 d^{2}=-1
$$

which is well known to be related to continued fractions (as studied in the author's Ph.D. thesis).

This equation has solutions given by the odd powers of $1+\sqrt{2}$, writing them as $k+d \sqrt{2}$. For example, these are the first three solutions:

$$
\begin{gathered}
k=1, d=1 \rightarrow 1^{2}+0^{2}=1^{2} ; \\
k=7, d=5 \rightarrow 3^{2}+4^{2}=5^{2} ; \\
k=41, d=29 \rightarrow 20^{2}+21^{2}=29^{2} .
\end{gathered}
$$

As we can see, $k / d$ is also an approximation given by the continued fraction expansion of $\sqrt{2}$ (the first, the third, etc.). This is not random, as continued fractions are well-known to be strictly related to Pell's equations (one can see the author's Ph.D. thesis for a detailed summary of this connection). 
Remark 3.1. One may also try to get approximations of an isosceles right triangle by giving up another condition, i.e., integrality of the hypothenuse. In this case, the Pell's equation gives $2 l^{2}-d^{2}= \pm 1$, and the solutions are given again (this time without any parity restriction) by the continued fraction approximants $d / l$ of $\sqrt{2}$.

\section{Heron's algorithm and continued fractions}

The algorithm of Heron, a particular case of Newton's method for finding approximated solutions to an equation, is strictly tied to continued fractions in some particular case.

We recall the following definition which uses elementary basic results (not proven here):

Definition 4.1 (Heron's algorithm). Let $x \in \mathbb{Q}_{a}$ be an absolute rational number which is not the square of another rational number. Let $a_{0}>\sqrt{x}$ be an approximation of the latter expression, which we suppose not to be already rational (so $x$ is not a square). We obtain then by recursion the following sequences:

$$
\begin{aligned}
b_{i} & =\frac{x}{a_{i}}, \\
a_{i+1} & =\frac{a_{i}+b_{i}}{2} .
\end{aligned}
$$

Notice that the $b_{i}$ converge to $\sqrt{x}$ from below and the $a_{i}$ converge to $\sqrt{x}$ from above. Then the intervals $\left[b_{i}, a_{i}\right]$ have as intersection exactly $\{\sqrt{x}\} \subset \mathbb{R}$. We say that $\left\{a_{i}\right\}_{i \in \mathbb{N}}$ is the Heron's algorithm sequence obtained starting with $a_{0}$. We may also accept $a_{0}<\sqrt{x}$, in which case the first couple $\left(a_{0}, b_{0}\right)$ is just swapped and the rest follows smoothly in the same way. Specifically, we will take $a_{0}=\lfloor\sqrt{x}\rfloor$.

Remark 4.2 (Matricial Heron's algorithm). Let $A_{0}=\left(\begin{array}{cc}a_{0} & x \\ 1 & a_{0}\end{array}\right)$, and consider the recursion:

$$
A_{i+1}=A_{i}^{2}
$$

Then one can prove by induction and direct calculation that $A_{i}$ must have the form $\left(\begin{array}{cc}n & x d \\ d & n\end{array}\right)$ for all $i \in \mathbb{N}$, where moreover we get the usual Heron's sequences from the columns of these matrices:

$$
\begin{aligned}
& a_{i}=A_{i}\left(\begin{array}{l}
1 \\
0
\end{array}\right)=\frac{n}{d} \\
& b_{i}=A_{i}\left(\begin{array}{l}
0 \\
1
\end{array}\right)=\frac{x d}{n}
\end{aligned}
$$

Remark 4.3 (Preliminary notations). We use the following notations:

- we write $\xi:=\sqrt{x}$;

- we write $\xi_{j}$ for the $j$-th approximant $\left[n_{0}, \ldots, n_{j-1}\right]$ of $\xi$; 
- let $\psi$ be the real number $n_{0}+\xi$, which has purely periodic continued fraction (see [4]) $\left[2 n_{0}, n_{1}, \ldots\right]$;

- we write $\psi_{j}$ for the $j$-th approximant $\left[2 n_{0}, \ldots, n_{j-1}\right]$ of $\psi$;

- let $c_{i}:=a_{0}+a_{i}$;

- let $S=\hat{0}$ be the swap matrix $\left(\begin{array}{ll}0 & 1 \\ 1 & 0\end{array}\right)$ (related to taking reciprocal in $\mathbb{P}_{1}$ );

- let $T_{n}$ be the translation matrix $\left(\begin{array}{ll}1 & n \\ 0 & 1\end{array}\right)$ (related to adding in $\mathbb{P}_{1}$ );

- let $M_{j}:=\hat{n}_{0} \cdot \hat{n}_{1} \cdots \hat{n}_{j-1}$, i.e. the matrix associated to $\xi_{j}$;

- let $M_{j}^{\prime}:=T_{n_{0}} \cdot M_{j}=T_{n_{0}} \cdot T_{n_{0}} \cdot S \cdot T_{n_{1}} \cdot S \cdots T_{n_{j}} \cdot S=T_{2 n_{0}} \cdot S \cdot T_{n_{1}} \cdot S \cdots T_{n_{j}} \cdot S=$ $\widehat{2 n_{0}} \cdot \hat{n}_{1} \cdots \hat{n}_{j}$, i.e. the matrix associated to $\psi_{j}$.

Notice that $\widehat{n_{j}}=T_{n_{j}} \cdot S=T_{1}^{n_{j}} \cdot S$.

We may prove the following:

Main Theorem 4.4. Suppose $\xi$ has continued fraction expansion $\left[n_{0}=\lfloor\sqrt{x}\rfloor, n_{1}, n_{2}, \ldots\right]$ with period of length 1 or 2. Apply Heron's algorithm to $a_{0}:=n_{0}$ obtaining a sequence $\left\{a_{0}, a_{1}, \ldots\right\}$. Then $a_{i}$ is the $2^{i}$-th approximant via the continued fraction. Vice versa, if the continued fraction has period length greater than 3, applying in the same way Heron's algorithm does not give the same sequence of approximants.

Proof 4.4. The case $i=0$ is already true by hypothesis. From the period assumption, we have for some $\lambda \in \mathbb{N}$ the following improper ${ }^{2}$ continued fraction:

$$
\xi=\left[\overline{a_{0}, \lambda, a_{0}, 0}\right]=a_{0}+\frac{1}{\lambda+\frac{1}{a_{0}+\xi}} .
$$

By elementary calculations this gives the equality ${ }^{3} \lambda=\frac{2 a_{0}}{x-a_{0}^{2}}$. Thus the case $i=1$ follows from the following calculation:

$$
\xi_{2}=a_{0}+\frac{1}{\lambda}=\frac{2 a_{0}^{2}}{2 a_{0}}+\frac{x-a_{0}^{2}}{2 a_{0}}=\frac{a_{0}}{2}+\frac{x}{2 a_{0}}=a_{1} .
$$

Moreover, $M:=\hat{a}_{0} \cdot \hat{\lambda} \cdot \hat{a}_{0}$ is classically known ${ }^{4}$ to be a symmetric matrix of the form $\left(\begin{array}{cc}x d & n \\ n & d\end{array}\right)$, with $\frac{n}{d}=\left[a_{0}, \lambda\right]=\xi_{2}$, as already observed in Remark 2.1. Then, with the notations for matricial Heron's algorithm, we have:

$$
A_{1}=M \cdot S=\hat{a}_{0} \cdot \hat{\lambda} \cdot \hat{a}_{0} \cdot \hat{0}
$$

\footnotetext{
${ }^{2}$ Proper continued fractions have nonzero digits; notice that in this case $\left[a_{0}, 0, y\right]=a_{0}+\frac{1}{0+1 / y}=a_{0}+y$.

${ }^{3}$ As a side note, we observe that the period length is 1 exactly when $x-a_{0}^{2}=1$, i.e., when $x$ is the successor of a square.

${ }^{4}$ As any palindrome product of symmetric matrices would be.
} 
Then we know that $A_{i}=(M \cdot S)^{2^{i-1}}$ for all $i \in \mathbb{N}$, but the latter is just the matrix $M_{2^{i}} \cdot T_{a_{0}}$, whose first column is the same as $M_{2^{i}}$. First column of $A_{i}$ represents $a_{i} \in \mathbb{P}^{1}$, first column of $M_{j}$ represents $\xi_{j} \in \mathbb{P}^{1}$, thus we have $a_{i}=\xi_{2^{i}}$, proving the direct theorem.

One could also take the matrices:

$$
\begin{aligned}
C_{1} & =\widehat{2 a_{0}} \cdot \widehat{\lambda} ; \\
C_{i+1} & =C_{i}^{2} .
\end{aligned}
$$

whose first columns represent ${ }^{5} c_{i} \in \mathbb{P}^{1}$. In this case, one gets an alternative proof observing that $C_{i}=M_{2^{i}}^{\prime}$ and deducing $c_{i}=\psi_{2^{i}}$.

For the vice versa, the alternative proof is the easiest to turn over: we see that if $c_{1}=[\alpha, \beta]$ and $c_{2}=[\alpha, \beta, \gamma, \delta]$ we must have that:

$$
\widehat{\alpha} \cdot \widehat{\beta} \cdot \widehat{\alpha} \cdot \widehat{\beta}=(\widehat{\alpha} \cdot \widehat{\beta})^{2}=\widehat{\alpha} \cdot \widehat{\beta} \cdot \widehat{\gamma} \cdot \widehat{\delta}
$$

that is, for the uniqueness of continued fractions, $\alpha=\gamma$ and $\beta=\delta$; in a similar way all even digits of the continued fraction of $\psi$ are $\alpha$ and all odd ones are $\beta$, thus the period length is necessarily a divisor of 2 .

Let us see some simple examples:

Example 4.5 (Period length 1). Approximating $\xi=\sqrt{2}$, we have:

$$
\begin{aligned}
& a_{0}=1 ; \\
& a_{1}=3 / 2=[1,2] ; \\
& a_{2}=17 / 12=[1,2,2,2] ; \\
& a_{3}=577 / 408=[1,2,2,2,2,2,2,2] ;
\end{aligned}
$$

Indeed, $\xi=[1, \overline{2}]$ and theorem's thesis holds in this case.

Example 4.6 (Period length 2). Approximating $\xi=\sqrt{6}$, we have:

$$
\begin{aligned}
& a_{0}=2 ; \\
& a_{1}=5 / 2=[2,2] ; \\
& a_{2}=49 / 20=[2,2,4,2] ; \\
& a_{3}=4801 / 1960=[2,2,4,2,4,2,4,2] ;
\end{aligned}
$$

Indeed, $\xi=[2, \overline{2,4}]$ and theorem's thesis holds in this case.

\footnotetext{
${ }^{5}$ This fact follows from the identity $C_{i}=T_{a_{0}} \cdot A_{i} \cdot T_{-a_{0}}$, where the last multiplication does not change the first column, while the first translates by $+a_{0}$, i.e. sends $a_{i} \in \mathbb{P}^{1}$ to $c_{i} \in \mathbb{P}^{1}$.
} 
Example 4.7 (Period length $>2$ ). Approximating $\xi=\sqrt{7}$, we have:

$$
\begin{aligned}
& a_{0}=2 ; \\
& a_{1}=11 / 4=[2,1,3] ; \\
& a_{2}=233 / 88=[2,1,1,1,5,5] ;
\end{aligned}
$$

Indeed, $\xi=[2, \overline{1,1,1,4}]$ and theorem's thesis does not hold in this case, confirming the necessity of the period length condition.

\section{Appendix A: Linear Fractional Transformations (LFTs) and projectivities}

We recall that the projective line $\mathbb{P}^{1}=\mathbb{P}^{1}(\mathbb{F})$ over a given base field $\mathbb{F}$ (usually the field of real numbers $\mathbb{R}$ ) is constructed associating to any non-zero vector $\left(\begin{array}{l}x \\ y\end{array}\right)$ in $\mathbb{F}^{2}$ the ratio $x / y$, possibly $\infty$ when denominator is zero, of its coordinates; thus we add a point to the usual line, and any point of this completed line is associated with an equivalency class of non-zero vectors of the plane by means of proportionality.

We say that two invertible matrices with entries in the given base field $\mathbb{F}$ are (projectively) equivalent when they are proportional:

$$
\left(\begin{array}{ll}
a & b \\
c & d
\end{array}\right) \cong\left(\begin{array}{ll}
\lambda a & \lambda b \\
\lambda c & \lambda d
\end{array}\right)
$$

and we will use the expression "homogeneous matrix" to describe an equivalence class of such matrices; when a homogeneous matrix has a non-zero determinant, it gives a transformation called projectivity by applying it to a point of the projective line considered as a vector: changing a representative matrix with a proportional one, the ratio of the resulting vector's coordinates is preserved, thus the transformation is well-defined.

Considering only the finite part of the line, projectivities give rise to the group of Linear Fractional Transformations (LFTs), that is the following applied to the field element $\left(\begin{array}{l}x \\ 1\end{array}\right)$ :

$$
f(x)=\frac{a x+b}{c x+d}
$$

which is defined everywhere with the exception of at most one point (the value for which the denominator vanishes), sent to infinity by the associated projectivity.

In this paper we use extensively these definitions, observing that adding a "digit" at the beginning of a continued fraction expansion has the effect of applying a projectivity/LFT to the number represented by the expansion and exploiting this fact in order to have cleaner proofs.

\section{Acknowledgements}

My most sincere thanks will go to my parents for supporting me as much as they could and to professor R. Dvornicich for giving me a great help in all my mathematical studies; I also have to 
thank all the other professors which formed my mathematical culture, among others my advisor

O. Rizzo for the teaching habilitation course during which I had most of the ideas for this paper to connect with more elementary mathematics.

\section{References}

[1] Cassels, J. W. S. (1957) An Introduction to Diophantine Approximation, Cambridge University Press, New York.

[2] Garver, R. (1932) A Square Root Method and Continued Fractions, The American Mathematical Monthly, 39 (9), 533-535.

[3] O'Dorney, E. (2015) Continued fractions and linear fractional transformations, Integers, 15, $1-23$.

[4] Perron, O. (1913) Die Lehre von den Kettenbrchen, B. G. Teubner, Lepizig, Berlin. 